\title{
Response to comment by Stuart Macgregor
}

\author{
Angelica Ronald • Lee M. Butcher • Sophia J. Docherty • \\ Oliver S. P. Davis · Leonard C. Schalkwyk • \\ Ian W. Craig $\cdot$ Robert Plomin
}

Published online: 13 December 2009

(C) Springer Science+Business Media, LLC 2009

We are grateful to Stuart Macgregor for his comment on our paper. We agree with his caveats about our work, which are mostly related to the selection criteria that we used to select SNPs from the first stage (DNA pooling) of our study. We should have used his method (Macgregor et al. 2006) for correcting mean allele frequency differences between high and low pools, perhaps in addition to our ad hoc multiple-criteria selection strategy which goes beyond statistical significance to take into account other criteria such as allele frequencies (see our Discussion). However, we could not go back and do the study again because the 42 SNPs nominated in our first stage (DNA pooling) were already individually genotyped in our independent replication sample.

Stuart Macgregor's comment raises more general issues. The speed of change in GWA analysis strategies is faster than the rate at which studies can be conducted and published. Moreover, GWA is still relatively new and therefore it should be accepted that we have not yet reached the stage at which the optimal strategy is obvious. The first GWA studies (WTCCC 2007) aimed for a single study that would yield definitive associations showing genome-wide significance. However, the field has shifted towards multiple-stage strategies like ours that attempt to beat the multiple-testing

This is a response to doi:10.1007/s10519-009-9313-9.

A. Ronald - L. M. Butcher - S. J. Docherty

O. S. P. Davis - L. C. Schalkwyk - I. W. Craig · R. Plomin Social Genetic and Developmental Psychiatry Centre, Institute of Psychiatry, De Crespigny Park, London SE5 8AF, UK

A. Ronald ( $\square)$

Centre for Brain and Cognitive Development, Birkbeck College,

Malet Street, London WC1E 7HX, UK

e-mail: a.ronald@bbk.ac.uk problem by winnowing the hundreds of thousands of SNPs in successive screening stages.

We would also like to highlight three features that we hope redeem our report despite its failure to find significant associations. First, DNA pooling will continue to have a role for screening as bigger and better samples and new DNA variants become available; even though the cost of arrays continues to decline, there are limits as to how much can be spent on individual genotyping. Second, quantitative genetic findings can be useful in guiding GWA; our research showing that the social and non-social components of autism spectrum disorder (ASD) differ genetically is a good example. (Case-control studies of diagnosed ASD conflate these two genetically heterogeneous components of ASD.) Third, our multiple-stage design set the bar very high for successful replication: SNPs that showed allele frequency differences between high versus low ASDsymptom groups in Stage 1 were then required to show significant associations for individual differences in autistic quantitative traits throughout the normal distribution; surviving SNPs then were required to show transmission disequilibrium in families with diagnosed ASD. With this ambitious design, any SNPs that survived these hurdles would have been especially exciting.

\section{Reference}

Macgregor S, Visscher PM, Montgomery G (2006) Analysis of pooled DNA samples on high density arrays without prior knowledge of differential hybridization rates. Nucleic Acids Res 34(7):e55

Wellcome Trust Case Control Consortium (2007) Genome-wide association study of 14,000 cases of seven common diseases and 3,000 shared controls. Nature 447:661-678 